\title{
Small Symmetrical Deformation of Thin Torus with Circular Cross-Section*
}

\author{
Bo-Hua Sun $^{1}$ \\ ${ }^{1}$ School of Civil Engineering \& Institute of Mechanics and Technology, \\ $X i$ 'an University of Architecture and Technology, Xi'an 710055, China \\ sunbohua@xauat.edu.cn
}

\begin{abstract}
By introducing a variable transformation $\xi=\frac{1}{2}(\sin \theta+1)$, a complex-form ordinary differential equation (ODE) for the small symmetrical deformation of an elastic torus is successfully transformed into the well-known Heun's ODE, whose exact solution is obtained in terms of Heun's functions. To overcome the computational difficulties of the complex-form ODE in dealing with boundary conditions, a real-form ODE system is proposed. A general code of numerical solution of the real-form ODE is written by using Maple. Some numerical studies are carried out and verified by finite element analysis. Our investigations show that the mechanics of an elastic torus are sensitive to the radius ratio, and suggest that the analysis of a torus should be done by using the bending theory of a shell. A general Maple code is provided as essential part of this paper.
\end{abstract}

\section{INTRODUCTION}

The torus or toroidal shell, in full or partial geometric form, is widely used in structural engineering. Among regular shells, such as circular cylindrical shells, conical shells, spherical shells, and tori, the deformation of the torus is one of the most difficulty topics due to its complicated topology.

The source of the difficulty comes from a geometric feature of the torus, whose Gauss curvature, $K=\frac{\sin \theta}{a(R+a \sin \theta)}$, changes its sign as principal radius of curvature $R_{\theta}$ when the angle $\theta$ goes from 0 to $2 \pi$. This means that the Gauss curvature has a turning point, $K=0$ at $\theta= \pm \pi$. The geometry of the torus surface is elliptic in $\theta \in[0, \pi]$, parabolic at $\theta=0$, and hyperbolic in $\theta \in[0,-\pi]$. Recall that the partial differential equations governing the elasticity of elliptic shells $K>0$ are themselves elliptic, while those for hyperbolic shells $K<0$ are hyperbolic. This means that the equations for a toroidal shell are of a mixed type, i.e., elliptic in the outer half and hyperbolic in the inner half. The existence of a turning point in a complete torus is one source of difficulty in finding a solution. Owing to the difficulty of solving differential equations with both hyperbolic and elliptic regions [17-21], various proposed asymptotic solutions have sigularity problem at the turning point of the Gauss curvature, where the Gauss curvature is zero $[17,18,20,21]$.

The torus has been studied for more than 100 years [16, 22], and various aspects have been extensively investigated. In this paper, we will restrict ourselves to the small symmetrical deformation of a torus with a circular cross-section, and with an emphasis on its theoretical formulation and the associated analytical and numerical solutions. We will not discuss topics such as non-circular cross-section, buckling, vibration, membrane solutions, and finite element analysis, which can be seen in the literature [27-30]. When the torus was first studied, high-order and complicated governing equations of a torus under symmetric loads were reduced to a single lower-order, complex-form ordinary differential equation (ODE) by Hans Reissner (1912)[2] when he was a professor at ETH in Switzerland. His colleague at ETH, Meissner [3], derived a complex-form equations for the shell of revolution. Hence, the first complex-form equation of the shells of revolution including torus is called the Reissner-Meissner equation. Reissner supervised a doctoral candidate, Gustav Weihs [1], who was the first person to receive a Dr.-Ing. in the field of torus research, in 1911. Unfortunatelly, I have not been able to find Weihs' thesis, and became familiar with his work from a citation by Wissler in his dissertation [4]. Wissler was supervised by Meissner, and was the second person to receive a Dr.-Ing in the field of torus research. The first exact series solution of the complex-form equation was obtained by Wissler (1916) [4], but his work had no followers, perhaps because his series solution was not linked to any special functions.

In 1912, Hans Reissner took a professor position at TU Berlin and brought torus research there. Under Reissner's influence, his younger colleague, F. Tölke, made some simplifications and derived a simpler complex-form equation of torus [5]. W. Chang (W. Zhang), the current writer's post-doctoral supervisor at Tsinghua University during 1989-1991, was Tölke's doctoral Mitarbeiter, who proposed an asymptotic solution to Tölke's equation for a large radius ratio $\alpha=a / R$ of the torus. Zhang's solution was the first successful asymptotic solution valid for the large ratio in terms of Bessel functions [6], and he received his Dr.-Ing. in 1944 from TU Berlin. In 1949, Hans Reissner's son Eric Resinner researched the pure bending of curved tubes [7], and Clark and Reissner published two papers [8, 9] on the asymptotic solution and calculations. In 1953, Dahl studied toroidal-shell expansion joints [10]. In 1959, Novozhilov published his monograph on the complex-form theory of shells, and gave an asymptotic solution [11] to the symmetrical deformation of a torus. In 1959, Tao introduced a variable transformation and successfully transformed the complex-form equation of a torus to a Heun-type ODE, and was the first person to find an exact solution that can be expressed in terms of Heun functions [12]. However, Tao did not perform any numerical calculations

\footnotetext{
* revision for Thin-Walled Structures, Manuscript number: TWST-D-20-00427R1
} 
using his exact solution. Nevertheless, both Wissler and Tao reached the peak in the research of a torus with small deformation. In 1965, Steele investigated toroidal pressure vessels, and was able to make a concise comparison of the volume and weight properties of enough shapes to provide a convenient basis for their design. Qian and Liang [14], Xia and Qian [15], and Zhang and Zhang [17, 18] conducted further study, sought an enhanced asymptotic solution valid for the full domain of $\theta \in[0,2 \pi]$. Sun $[20,21,23]$ was the first person to derive displacement-type equations of a torus and proposed a closed-form solution when the radius ratio tends to be null. The first monograph on torus was edited by Sun and published in 2012 [21].

In 1959 Novozhilov systematiclly developed a comprehensive complex-form theory of thin shells and reformulated the symmetrical deformation of a torus [11], then he proposed an asymptotic solution for the thin torus [11]. However, no exact solution in terms of special functions has been obtained for Novozhilov's complex-form ODE of symmetrical deformation of a torus. We will shoulder this burden and propose such a solution. Once we have the exact solution, the convergence over the full domain of $\theta \in[0,2 \pi]$ can then be guaranteed.

The remainder of this paper is organized as follows. Section 2 presents a formulation of the Novzhilov complex-form ordinary differential equation of a torus. Section 3 introduces a variable transformation and finds an exact solution of the complex ODE. Section 4 splits the complex-ODE into a real-form ODE. In Section 5, we carry out numerical simulations for three cases by using our own Maple code. Section 6 discusses the difference between the membrane and bending theory of a torus. Section 7 verifies our solution by finite element analysis. Section 8 provides conclusions and recommendations.

\section{NOVOZHILOV'S FORMULATION OF SYMMETRICAL DEFORMATION OF A TORUS}

For the torus shown in Fig. 1, the positions of points on the middle surface will be determined by the angles $\theta$ and $\varphi$. Further, let $R_{1}$ be the radius of curvature of the meridian and $R_{2}$ the radius of curvature of the normal section, tangential to the parallel circle. This second radius is equal to the segment of the perpendicular to the middle surface between this surface and the axis of the torus.

The principal radii of curvature are given by

$$
R_{1}=a, R_{2}=R \frac{1+\alpha \sin \theta}{\sin \theta}
$$

where $\alpha=a / R$.

The Lamé parameters in this case are determined by the expressions

$$
A_{1}=R_{1}=a, A_{2}=R_{2} \sin \theta=R+a \sin \theta .
$$
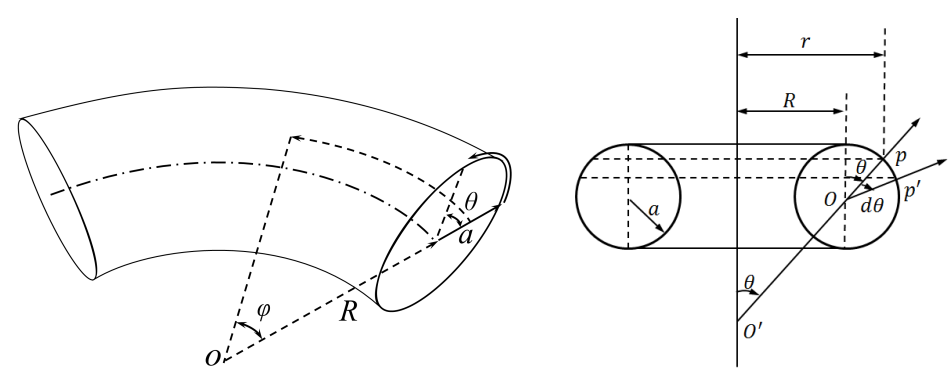

Figure 1: Torus and cross-sectional view. Torus and geometry. Principal radii of curvature are $R_{1}=a$ and $R_{2}=a+\frac{R}{\sin \theta}$; principal curvature $K_{1}=\frac{1}{a}, K_{2}=\frac{\sin \theta}{R+a \sin \theta} ;$ Gauss curvature $K=K_{1} K_{2}=\frac{\sin \theta}{a(R+a \sin \theta)}$.

The complex-form governing equation of a torus for symmetrical deformation was obtained by Novozhilov (1959) [11] as follows:

$$
(1+\alpha \sin \theta) \frac{d^{2} V}{d \theta^{2}}-\alpha \cos \theta \frac{d V}{d \theta}+2 i d^{2} \sin \theta V=P(\theta)
$$


Eq. 3 is called the Novozhilov equation of a torus. In which, $V(\theta)$ is a auxiliary complex function of real variable $\theta$ and the right hand term is

$$
\begin{aligned}
P(\theta) & =-2 d^{2}\left(2 d^{2} C+\frac{1}{2} i \alpha q a\right) \cos \theta, \\
2 d^{2} & =\frac{a^{2}}{R h} \sqrt{12\left(1-\mu^{2}\right)}
\end{aligned}
$$

where $\mu$ is the Poisson ratio, $h$ is the thickness, $q$ is the distributed load, and $C$ is an integration constant that can be determined from static considerations. The analysis of the torus has thus been reduced to the problem of finding a solution for the ODE in (3).

Having the auxiliary function $V(\theta)$, all other quantities can be expressed in terms of $V(\theta)$. The middle surface resultant forces are

$$
\begin{aligned}
& T_{1}=-\frac{\alpha \cos \theta}{2 d^{2}(1+\alpha \sin \theta)} \operatorname{Im}(V)+\frac{q a}{2} \frac{2+\alpha \sin \theta}{1+\alpha \sin \theta}-\alpha C \frac{\alpha+\sin \theta}{(1+\alpha \sin \theta)^{2}} \\
& T_{2}=-\frac{1}{2 d^{2}} \operatorname{Im}\left[\frac{d}{d \theta}\left(\frac{V}{1+\alpha \sin \theta}\right)\right]+\frac{q a}{2}+\alpha C \frac{\alpha+\sin \theta}{(1+\alpha \sin \theta)^{2}}
\end{aligned}
$$

where the $R e$ and $I m$ stand for the real and imaginary portion of the complex function $V(\theta)$. And resultant moments are

$$
\begin{aligned}
& M_{1}=-\frac{h}{2 d^{2} \sqrt{12\left(1-\mu^{2}\right)}}\left\{\frac{\mu \alpha \cos \theta}{(1+\alpha \sin \theta)^{2}} \operatorname{Re}(V)+R e\left[\frac{d}{d \theta}\left(\frac{V}{1+\alpha \sin \theta}\right)\right]\right\}, \\
& M_{2}=-\frac{h}{2 d^{2} \sqrt{12\left(1-\mu^{2}\right)}}\left\{\frac{\alpha \cos \theta}{(1+\alpha \sin \theta)^{2}} \operatorname{Re}(V)+\mu R e\left[\frac{d}{d \theta}\left(\frac{\mu V}{1+\alpha \sin \theta}\right)\right]\right\},
\end{aligned}
$$

resultant shear force is

$$
N_{1}=-\frac{h}{a \sqrt{12\left(1-\mu^{2}\right)}} \frac{\sin \theta \operatorname{Im}(V)+2 d^{2} C \cos \theta}{(1+\alpha \sin \theta)^{2}},
$$

and angle of rotation of the tangent to the meridian is

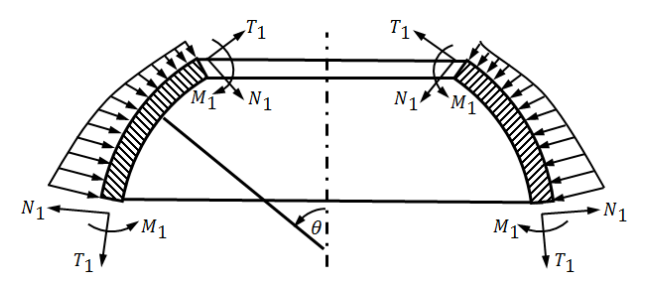

Figure 2: Components of forces and moments on middle surface

$$
\vartheta=-\frac{1}{E h} \frac{1}{\alpha(1+\alpha \sin \theta)} \operatorname{Re}(V) .
$$

The component of the displacement of an arbitrary point on the meridian in the direction of the axis of the torus is

$$
\triangle_{z}=-a \int_{0}^{\theta} \vartheta \cos \theta d \theta+\triangle_{z}(0)
$$

and the component of the displacement in the direction perpendicular to this axis is

$$
\triangle_{x}=\frac{R}{E h}(1+\alpha \sin \theta)\left(T_{2}-\mu T_{1}\right) .
$$

The deformation displacement components of the middle surface in Fig. 3 can be obtained as

$$
\begin{aligned}
u & =\triangle_{x} \cos \theta-\triangle_{z} \sin \theta \\
w & =\triangle_{x} \sin \theta+\triangle_{z} \cos \theta
\end{aligned}
$$




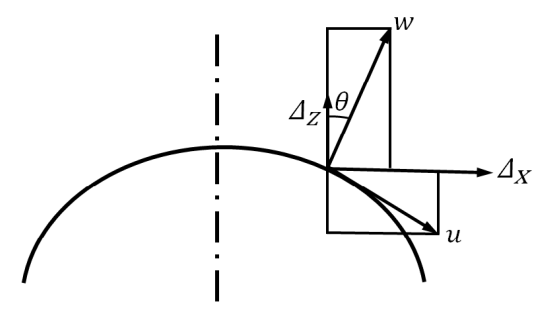

Figure 3: $u$ and $w$ are the components of displacement on the middle surface

The components of the middle surface forces are

$$
\begin{aligned}
& Q_{x}=T_{1} \cos \theta+N_{1} \sin \theta \\
& Q_{z}=T_{1} \sin \theta-N_{1} \cos \theta
\end{aligned}
$$

where $Q_{z}$ and $Q_{x}$, respectively, are the components of the forces in the direction of the axis of the torus and perpendicular to this axis, which act through the contemplated point of the meridian, as shown in Fig. 4.

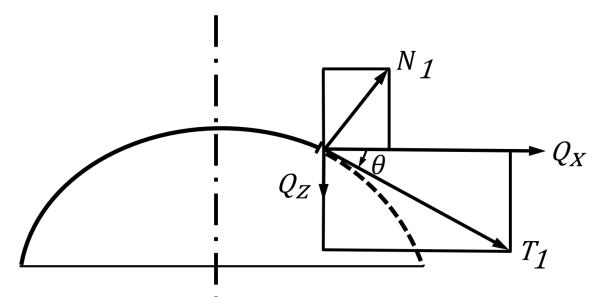

Figure 4: $Q_{z}$ and $Q_{x}$ are the components of the forces on the middle surface

\section{EXACT SOLUTION OF SMALL SYMMETRICAL DEFORMATION OF TORUS}

To solve Eq. 3, Wissler [4] introduced a variable transformation,

$$
x=\sin \theta,
$$

which leads to $d x=\cos \theta d \theta, \frac{d V}{d \theta}=\frac{d V}{d x} \frac{d x}{d \theta}=\cos \theta \frac{d V}{d x}$, and $\frac{d^{2} V}{d \theta^{2}}=\cos ^{2} \theta \frac{d 2 V}{d x^{2}}-\sin \theta \frac{d V}{d x}=\left(1-x^{2}\right) \frac{d^{2} V}{d x 2}-x \frac{d V}{d x}$. Hence Eq. 3 is transformed as follows:

$$
\left(1-x^{2}\right)(1+\alpha x) \frac{d^{2} V}{d x^{2}}-(x+\alpha) \frac{d V}{d x}+2 i d^{2} x V=P(x)
$$

where $P(x)=-2 d^{2}\left(2 d^{2} C+\frac{1}{2} i \alpha q a\right) \sqrt{1-x^{2}}$.

Eq. 19 is a Fuchian-type differential equation whose series solution was given by Wissler [4], which is an exact solution but is not linked to any known functions.

To establish a relationship between Eq. 19 and well-known equations, let us introduce another variable transformation,

$$
\xi=\frac{1}{2} x+\frac{1}{2}=\frac{1}{2}(\sin \theta+1)
$$

Thus $\frac{d V}{d x}=\frac{1}{2} \frac{d V}{d \xi}, \frac{d^{2} V}{d x^{2}}=\frac{1}{4} \frac{d^{2} V}{d \xi^{2}}$, and $1-x^{2}=-4 \xi(\xi-1)$, and Eq. 19 can be written as

$$
\xi(\xi-1)\left[\xi-\left(\frac{1}{2}-\frac{1}{2 \alpha}\right)\right] \frac{d^{2} V}{d \xi^{2}}+\frac{1}{2 \alpha}\left(\xi-\frac{1}{2}+\frac{\alpha}{2}\right) \frac{d V}{d \xi}+\left(-\frac{4 i d^{2}}{\alpha} \xi+\frac{i d^{2}}{\alpha}\right) V=P(\xi),
$$

where $P(\xi)=\frac{2 d^{2}}{\alpha}\left(2 d^{2} C+\frac{1}{2} i \alpha q a\right) \sqrt{\xi(1-\xi)}$; another common form is

$$
\frac{d^{2} V}{d \xi^{2}}+\left(\frac{\frac{1}{2}}{\xi}+\frac{\frac{1}{2}}{\xi-1}+\frac{-1}{\xi-\frac{\alpha-1}{2 \alpha}}\right) \frac{d V}{d \xi}+\frac{-\frac{2 i d^{2}}{\alpha} \xi+\frac{i d^{2}}{\alpha}}{\xi(\xi-1)\left(\xi-\frac{\alpha-1}{2 \epsilon}\right)} V=f
$$


where

$$
f=\frac{2 d^{2}}{\alpha} \frac{1}{\xi(\xi-1)\left(\xi-\frac{\alpha-1}{2 \alpha}\right)}\left(2 d^{2} C+\frac{1}{2} i \alpha a q\right) .
$$

It is easy to see that Eq. 21 or 22 is a Fuchian-type differential equation with four regular singular points: $0,1, \frac{\alpha-1}{2 \epsilon}$, and $\infty$. Eq. 22 was studied by Heun (1889) [24, 25], whose solutions can be represented by Heun's functions. Named after Karl Heun (1859-1929), these are unique local Frobenius solutions of a second-order linear ordinary differential equation of the Fuchsian type, which in the general case has four regular singular points.

The standard Heun ordinary differential equation is

$$
\begin{aligned}
& \frac{d^{2} y(z)}{d z^{2}}+\frac{(\sigma+\beta+1) z^{2}+[(-\delta-\rho) c-\sigma+\delta-\beta-1] z+\rho c}{z(z-1)(z-c)} \frac{d y(z)}{d z} \\
& +\frac{(z \beta \sigma-p) y(z)}{z(z-1)(z-c)}=0 .
\end{aligned}
$$

Its solution is given by

$$
\begin{aligned}
y(z) & =C_{1} \operatorname{HeunG}(c, p, \beta, \sigma, \rho, \delta, z) \\
& +C_{2} z^{1-\rho} \operatorname{Heun} G(c, p-(-1+\rho)(\delta(-1+c)+\sigma+\beta-\rho+1), \\
& \beta+1-\rho, \sigma-\rho+1,-\rho+2, \delta, z),
\end{aligned}
$$

where $\operatorname{Heun} G(c, p, \beta, \sigma, \rho, \delta, z)$ is the Heun function, which can be expressed in series as

$$
\operatorname{Heun} G(c, p, \beta, \sigma, \rho, \delta, z)=1+\sigma \beta \sum_{n=1}^{\infty} \frac{G_{n}(p)}{n !(\rho)_{n}}\left(\frac{z}{c}\right)^{n},
$$

where

$$
\begin{aligned}
n ! & =n(n-1) \ldots 1, \\
(\rho)_{n} & =\rho(\rho+1)(\rho+2) \ldots(\rho+n-1), \\
G_{1}(p) & =p \\
G_{2}(p) & =\sigma \beta p^{2}+[(\beta+\sigma-\delta+1)+(\rho+\delta) c] p-\beta \rho, \\
G_{n+1}(p) & =\{n[(\sigma+\beta-\delta+n)+(\rho+\delta+n-1) c]+\sigma \beta p\} G_{n}(p) \\
& -(c+n-1)(\beta+n-1)(\rho+n-1) n c G_{n-1}(p) .
\end{aligned}
$$

Heun functions generalize the hypergeometric function. Because of their wide range of applications, they can be considered the 21st-century successors to hypergeometric functions [31]. The exact solution of Eq. 21 is the sum of a homogenous solution $V^{h}(x)$ and particular solution $V^{p}(x)$, i.e., $V=V^{h}+V^{p}$, both of which can be expressed by Heun functions. The homogenous solution can be given as

$$
V^{h}(x)=C_{1} y_{1}(x)+C_{2}(x+1)^{\frac{1}{2}} y_{2}(x)
$$

where

$$
\begin{aligned}
& y_{1}(x)=\operatorname{Heun} G\left(\frac{\alpha-1}{2 \alpha},-\frac{i d^{2}}{\alpha}, a_{1}, a_{2}, \frac{1}{2}, \frac{1}{2}, \frac{1}{2}(x+1)\right), \\
& y_{2}(x)=\operatorname{Heun} G\left(\frac{\alpha-1}{2 \alpha},-\frac{1}{8} \frac{8 i d^{2}+3 \alpha+1}{\alpha}, b_{1}, b_{2}, \frac{1}{2}, \frac{1}{2}(x+1)\right) \text {, }
\end{aligned}
$$

where

$$
\begin{aligned}
& a_{1}=-\frac{1}{2} \frac{\sqrt{\alpha} \sqrt{8 i d^{2}+\alpha}+\alpha}{\alpha}, \\
& a_{2}=\frac{1}{2} \frac{8 i d^{2}+\sqrt{\alpha} \sqrt{8 i d^{2}+\alpha}-\alpha}{\sqrt{\alpha} \sqrt{8 i d^{2}+\alpha}+2 \alpha}, \\
& b_{1}=-\frac{1}{2} \frac{\sqrt{8 i d^{2}+\alpha}}{\sqrt{\alpha}}, \\
& b_{2}=\frac{1}{2} \frac{8 i d^{2}+2 \sqrt{\alpha} \sqrt{8 i d^{2}+\alpha}+\alpha}{\sqrt{\alpha} \sqrt{8 i d^{2}+\alpha}+2 \alpha}, \frac{3}{2} .
\end{aligned}
$$


Substituting Wissler's transformation $x=\sin \theta$ into the above solutions, we have

$$
V^{h}(\theta)=C_{1} y_{1}(\theta)+C_{2}(\sin \theta+1)^{\frac{1}{2}} y_{2}(\theta)
$$

where

$$
\begin{aligned}
& y_{1}(\theta)=\operatorname{Heun} G\left(\frac{\alpha-1}{2 \alpha},-\frac{i d^{2}}{\alpha}, a_{1}, a_{2}, \frac{1}{2}, \frac{1}{2}, \frac{1}{2}(\sin \theta+1)\right), \\
& y_{2}(\theta)=\operatorname{Heun} G\left(\frac{\alpha-1}{2 \alpha},-\frac{1}{8} \frac{8 i d^{2}+3 \alpha+1}{\alpha}, b_{1}, b_{2}, \frac{1}{2}, \frac{1}{2}(\sin \theta+1)\right) .
\end{aligned}
$$

A particular solution $V^{p}$ can generally be expressed analytically by Heun functions, which we do not discuss here due to its complicated form. If the loading condition is not constant, then some integrations involving the Heun function in $V^{p}$ may not be obtainable analytically.

\section{THE SPLIT OF THE NOVOZHILOV EQUATION}

For further computation, the solution $V(\theta)$ must be split into real and imaginary parts, i.e., $\operatorname{Re}(V)$ and $\operatorname{Im}(V)$, respectively. However, this is not possible analytically due to the complicated expression of the Heun function. Without $\operatorname{Re}(V)$ and $\operatorname{Im}(V)$, no analytical components of force, moment, and displacement for the torus can be obtained, which limits the use of the complexform analytical solution obtained in the previous section. Therefore, it is natural to seek a numerical solution based on the Novozhilov equation, i.e., Eq. 3.

Let us write $V(\theta)$ as

$$
V(\theta)=A(\theta)+i B(\theta)
$$

with the imaginary $i=\sqrt{-1}$, where $A(\theta)=\operatorname{Re}(V)$ and $B(\theta)=\operatorname{Im}(V)$ are the real and imaginary parts of $V(\theta)$. Substituting Eq. 42 in Eq. 3, we obtain

$$
\begin{aligned}
& (1+\alpha \sin \theta) \frac{d^{2} A}{d \theta^{2}}-\alpha \cos \theta \frac{d A}{d \theta}-2 d^{2} \sin \theta B=-2 d^{2}\left(2 d^{2} C\right) \cos \theta \\
& (1+\alpha \sin \theta) \frac{d^{2} B}{d \theta^{2}}-\alpha \cos \theta \frac{d B}{d \theta}+2 i d^{2} \sin \theta A=-2 d^{2}\left(\frac{1}{2} \alpha q a\right) \cos \theta
\end{aligned}
$$

Because we cannot analytically represent the integration of the displacement component of an arbitrary point on the meridian in the direction of the axis of a torus in Eq. 12, we use the corresponding differential equation,

$$
\frac{d \triangle_{z}}{d \theta}-\frac{a}{E h} \frac{A \cos \theta}{\alpha(1+\alpha \sin \theta)}=0 .
$$

Now, the symmetrical deformation problem becomes one of finding the functions $A(\theta), B(\theta)$, and $\triangle_{z}(\theta)$.

With these functions, we can compute all other quantities, such as $T 1, T_{2}, M_{1}, M_{2}$ and $N_{1}$, as well as $u, w$ and $\vartheta$. These can be expressed in terms of $A(\theta)$ and $B(\theta)$ as follows

$$
\begin{aligned}
& T_{1}=-\frac{\alpha \cos \theta}{2 d^{2}(1+\alpha \sin \theta)} B(\theta)+\frac{q a}{2} \frac{2+\alpha \sin \theta}{1+\alpha \sin \theta}-\alpha C \frac{\alpha+\sin \theta}{(1+\alpha \sin \theta)^{2}}, \\
& T_{2}=-\frac{1}{2 d^{2}}\left[\frac{d}{d \theta}\left(\frac{B(\theta)}{1+\alpha \sin \theta}\right)\right]+\frac{q a}{2}+\alpha C \frac{\alpha+\sin \theta}{(1+\alpha \sin \theta)^{2}} .
\end{aligned}
$$

The resultant moments are

$$
\begin{aligned}
& M_{1}=-\frac{h}{2 d^{2} \sqrt{12\left(1-\mu^{2}\right)}}\left\{\frac{\mu \alpha \cos \theta}{(1+\alpha \sin \theta)^{2}} A(\theta)+\left[\frac{d}{d \theta}\left(\frac{A(\theta)}{1+\alpha \sin \theta}\right)\right]\right\} \\
& M_{2}=-\frac{h}{2 d^{2} \sqrt{12\left(1-\mu^{2}\right)}}\left\{\frac{\alpha \cos \theta}{(1+\alpha \sin \theta)^{2}} A(\theta)+\mu\left[\frac{d}{d \theta}\left(\frac{\mu A(\theta)}{1+\alpha \sin \theta}\right)\right]\right\},
\end{aligned}
$$

the resultant shear force is

$$
N_{1}=-\frac{h}{a \sqrt{12\left(1-\mu^{2}\right)}} \frac{\sin \theta B(\theta)+2 d^{2} C \cos \theta}{(1+\alpha \sin \theta)^{2}},
$$


and the angle of rotation of the tangent to the meridian is

$$
\vartheta=-\frac{1}{E h} \frac{A(\theta)}{\alpha(1+\alpha \sin \theta)} .
$$

The bending-related shell stresses are obtained by combining the direct stresses due to the stress resultants with the flexural stresses due to the bending moments,

$$
\sigma_{1}=\frac{T_{1}}{h} \pm \frac{6 M_{1}}{h^{2}}, \sigma_{2}=\frac{T_{2}}{h} \pm \frac{6 M_{2}}{h^{2}}
$$

The coupled differential equations (43) can be decoupled into differential equations about $A(\theta)$ and $B(\theta)$, both fourth-order ODEs, but no analytical solutions about $A(\theta)$ and $B(\theta)$ can be obtained.

\section{NUMERICAL STUDIES OF SYMMETRICAL DEFORMATION OF TORUS}

We numerically solve equations 43 and 44 for some typical cases. Unless otherwise stated, numerical calculations in this paper are based on the data in Table I.

We vary the radius $a=0.18 k, k=1,2,3,4,5$, while other quantities are unchanged. Table I lists the geometric and material properties of the torus.

Table I: Data of torus

\begin{tabular}{c|c|c|c|c|c|c|c}
\multicolumn{1}{c}{ Table I: Data of torus } \\
\hline Quantities & $R$ & $a$ & $h$ & $E$ & $\mu$ & $M_{0}$ & $Q_{0}$ \\
\hline Units & $\mathrm{m}$ & $\mathrm{m}$ & $\mathrm{m}$ & $N / m^{2}$ & 1 & $N$ & $N / m$ \\
\hline Data & 1 & $0.18 k$ & 0.04 & $2.07 \times 10^{11}$ & 0.3 & 1 & 1 \\
\hline
\end{tabular}

Note: Loads are set to unit values in all simulations. Since a small deformation is a linear problem, the superposition principle can be used with different loads, and solutions obtained by multiplying our results by an appropriate factor.

The geometry of these tori is shown in Fig. 5.

A. Complete torus with a penetrate cut along the parallel $\theta=\frac{\pi}{2}$ and loaded with distributed bending moment $Q_{0}$

For a complete torus with a penetrate cut along the parallel $\theta=\frac{\pi}{2}$ and loaded with distributed bending moment $Q_{0}$, the loading condition is shown in Fig. 6. The boundary condition is:

$$
\begin{gathered}
\theta=\frac{\pi}{2}: T_{1}=-Q_{0}, N_{1}=0, M_{1}=0, \\
\theta=-\frac{3 \pi}{2}: T_{1}=-Q_{0}, N_{1}=0, M_{1}=0, \\
\theta=-\frac{3 \pi}{2}: \triangle_{z}=0 .
\end{gathered}
$$

For this problem, the constant $C$ can be determined by the boundary condition of $T_{1}$ at $\theta=\frac{\pi}{2}$, namely

$$
\theta=\frac{\pi}{2}: T_{1}=-Q_{0}
$$

Taking into account the uniform distribution load $q=0$, the above equation gives $C=\frac{1+\alpha}{\alpha} Q_{0}$, and the boundary loading condition $T_{1}=-Q_{0}$ for both $\theta=\frac{\pi}{2}$ and $\theta=-\frac{3 \pi}{2}$ is satisfied. The rest of the boundary condition can be expressed in terms of $A(\theta)$ and $B(\theta)$ as

$$
\begin{gathered}
\theta=\frac{\pi}{2}: B=0, \frac{d A}{d \theta}=0 \\
\theta=-\frac{3 \pi}{2}: B=0, \frac{d A}{d \theta}=0 \\
\theta=-\frac{3 \pi}{2}: \triangle_{z}=0 .
\end{gathered}
$$



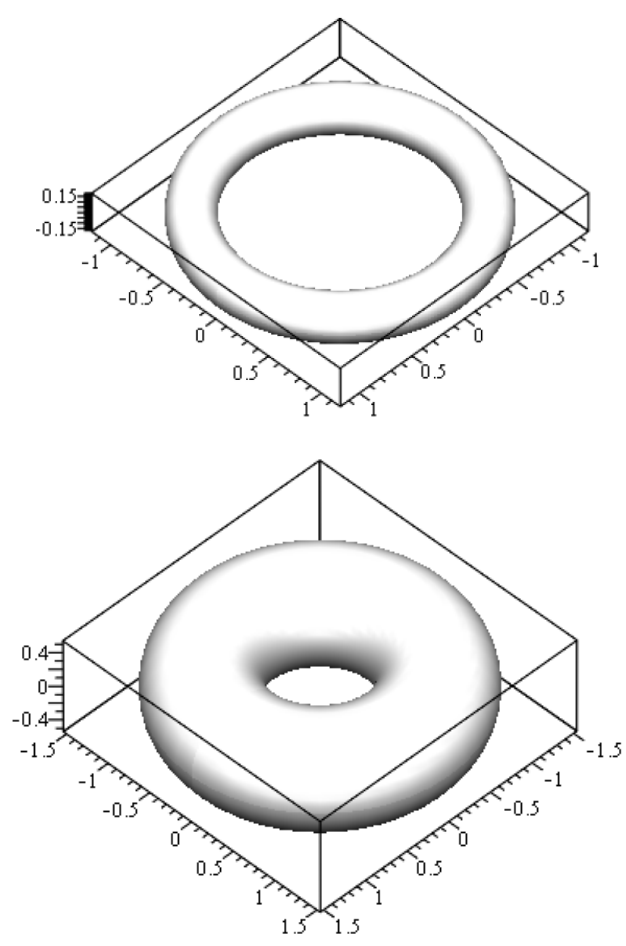

(a)

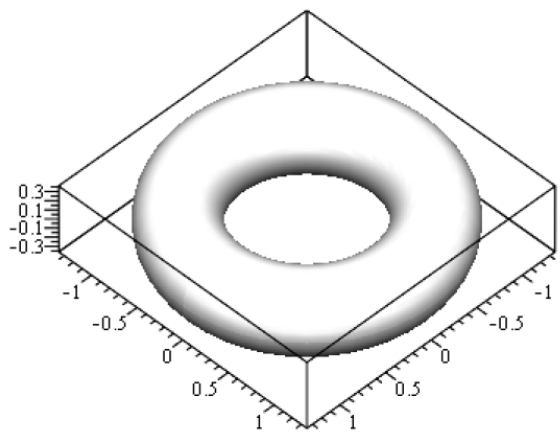

(b)

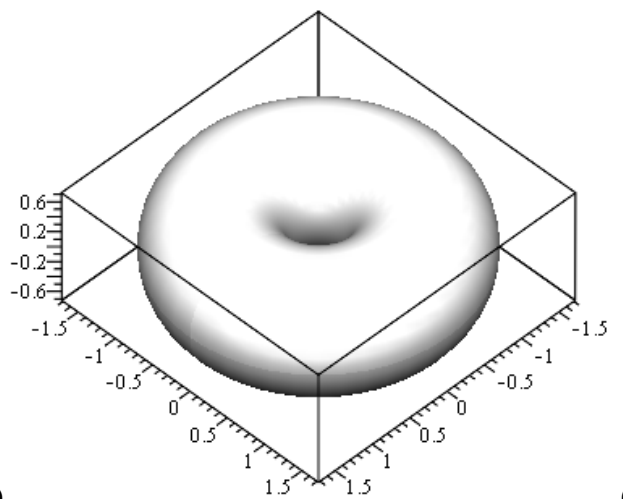

(d)

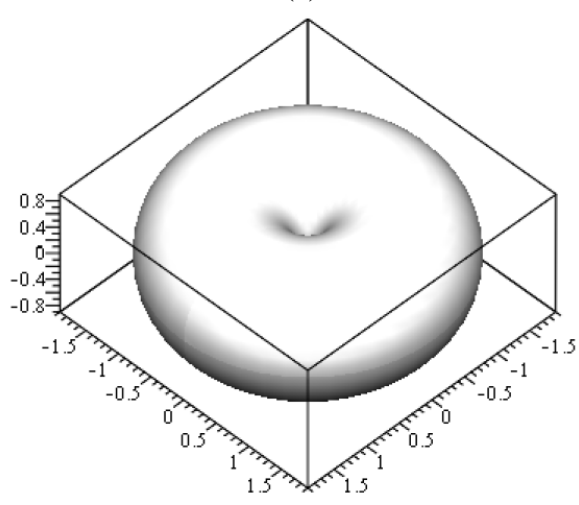

(e)

Figure 5: Torus geometry with different radii $a=0.18 k(k=1,2,3,4,5)$

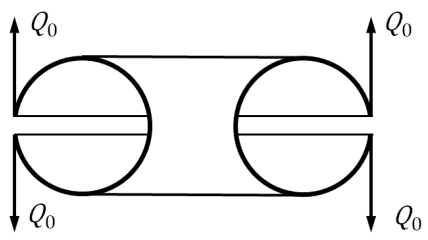

Figure 6: Torus with a cut along its parallel at $\theta=\frac{\pi}{2}$ or $\theta=-\frac{\pi}{2}$ under load $Q_{0}$

Now, the problem becomes to solve $A(\theta)$ and $B(\theta)$ under conditions (56).

A general code for this case is written by Maple and it will be valid for other caes if changing boundary conditions. For further investigation, it is our honor to provide the code below.

restart:

for $\mathrm{k}$ from 1 to 5 do:

$\mathrm{a}:=0.18 * \mathrm{k}$ :

$\mathrm{R}:=1$ :

alpha:=a/(R):

$\mathrm{h}:=0.04$ : 
$\mathrm{mu}:=0.3$ :

$\mathrm{E}:=2.07 \mathrm{e}+11$ :

$\mathrm{q}:=0$ :

$\mathrm{Q}:=1$ :

$\mathrm{C}:=(1+$ alpha $) /($ alpha $) * \mathrm{Q}$ :

$\mathrm{d}:=\operatorname{sqrt}(1 /(2) *(\operatorname{sqrt}(12 *(1-\mathrm{mu} * \mathrm{mu})) * \mathrm{a} * \mathrm{a}) /(\mathrm{R} * \mathrm{~h}))$ :

kappa: $=\operatorname{sqrt}\left(12 *\left(1-\mathrm{mu}^{*} \mathrm{mu}\right)\right)$ :

eta: $=1+$ alpha* $\sin ($ theta $)$ :

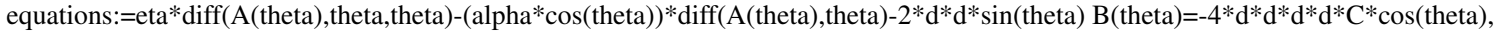
eta*diff(B(theta), theta,theta)-(alpha*cos(theta) $) * \operatorname{diff}(B($ theta $)$,theta $)+2 * d * d * \sin ($ theta $)$ A $($ theta $)=-2 * d * d *\left(\left(\right.\right.$ alpha* $\left.\left.\mathrm{q}^{*} \mathrm{a}\right) /(2)\right)$,

$\operatorname{diff}($ Delta $[\mathrm{z}]($ theta $)$, theta $)=(\mathrm{a}) /\left(\mathrm{E}^{*} \mathrm{~h}\right) *(\mathrm{~A}($ theta $)) /($ alpha*eta $) * \cos ($ theta $)$;

boundary: $=\mathrm{B}(-(3 * \mathrm{Pi}) /(2))=0, \mathrm{~B}(\mathrm{Pi} /(2))=0, \mathrm{D}(\mathrm{A})(-(3 * \mathrm{Pi}) /(2))=0, \mathrm{D}(\mathrm{A})(\mathrm{Pi} /(2))=0, \mathrm{Delta}[\mathrm{z}](-(3 * \mathrm{Pi}) /(2))=0$;

solution:=dsolve([equations, boundary],[A(theta),B(theta), Delta[z](theta)],numeric,

abserr $=0.001$,output $=$ listprocedure $)$ :

$\mathrm{T} 1[\mathrm{k}]:=-\mathrm{alpha} * \cos ($ theta $) * \mathrm{R} * \mathrm{~h} * \mathrm{rhs}(\operatorname{sol}[4]) /($ eta* $(\mathrm{a} * \mathrm{a} * \mathrm{kappa}))+(1 / 2) * \mathrm{q} * \mathrm{a} *(2+$ alpha* $\sin ($ theta $)) /$ eta-alpha* $\mathrm{C} *($ alpha+sin(theta $)) /($ eta*eta $)$;

$\mathrm{T} 2[\mathrm{k}]:=-\mathrm{R} * \mathrm{~h} *(\mathrm{rhs}(\operatorname{sol}[5]) /(\mathrm{eta} * \mathrm{kappa})+(\operatorname{diff}(1 / \mathrm{eta}$, theta)$) * \mathrm{rhs}(\operatorname{sol}[4])) /(\mathrm{a} * \mathrm{a})$

$+(1 / 2) * \mathrm{q} * \mathrm{a}+\mathrm{alph} * \mathrm{C} *($ alpha+sin(theta) $) /($ eta*eta $)$

$\mathrm{M} 1[\mathrm{k}]:=-\left(\mathrm{R} * \mathrm{~h} * \mathrm{~h} /\left(\mathrm{kappa}{ }^{*} \mathrm{kappa}\right)\right) *\left(\mathrm{mu}^{*} \mathrm{alpha} * \cos (\right.$ theta $) * \mathrm{rhs}(\operatorname{sol}[2]) /($ eta*eta $)+\mathrm{rhs}(\mathrm{sol}[3]) / \mathrm{eta}$

$+\operatorname{rhs}(\operatorname{sol}[2]) *(\operatorname{diff}(1 / \mathrm{eta}$, theta $))) /((\mathrm{a} * \mathrm{a}) *($ kappa*kappa $))$;

$\mathrm{M} 2[\mathrm{k}]:=-(\mathrm{R} * \mathrm{~h} * \mathrm{~h} /(\mathrm{kappa} * \mathrm{kappa}) *($ alpha*cos$($ theta $) * \mathrm{rhs}(\operatorname{sol}[2]) /($ eta*eta $)+\mathrm{mu} * \mathrm{rhs}(\mathrm{sol}[3]) / \mathrm{eta}$

$+\mathrm{mu}^{*} \mathrm{rhs}(\operatorname{sol}[2]) *(\operatorname{diff}(1 / \mathrm{eta}$, theta $\left.))\right) /(\mathrm{a} * \mathrm{a} * \mathrm{kappa} * \mathrm{kappa})$

$\mathrm{N} 1[\mathrm{k}]:=-\mathrm{h} * \sin ($ theta $) * \mathrm{rhs}(\operatorname{sol}[4]) /(\mathrm{a} * \mathrm{kappa} *($ eta*eta)$)-\mathrm{a} / \mathrm{R} * \mathrm{C} * \cos ($ theta $) /($ eta*eta);

vartheta[k] :=-rhs $(\operatorname{sol}[2]) /\left(\mathrm{E}^{*} \mathrm{~h} *\right.$ alpha*eta):

od;

The numerical results are shown in Fig. 7, 8, and 9, and the stress is shown in Fig. 10.
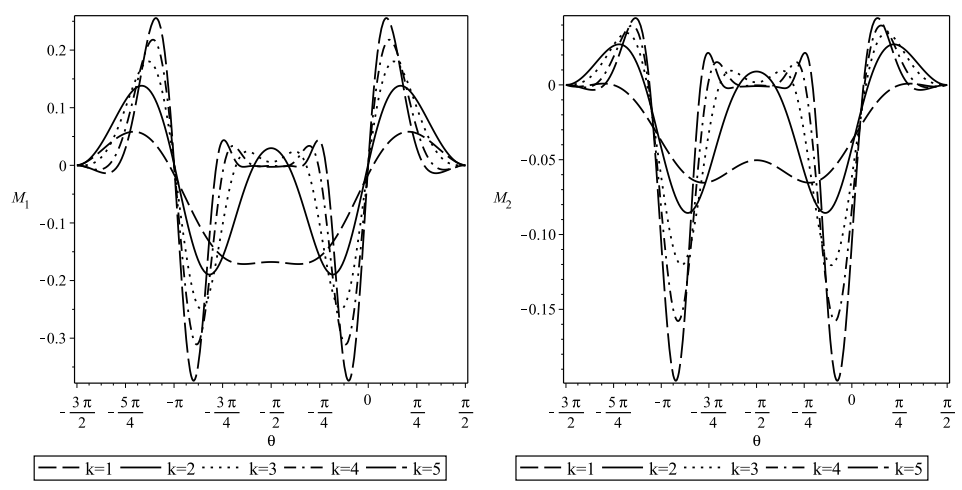

Figure 7: Left: Bending moment $M_{1}$; Right: Bending moment $M_{2}$. The torus radius is $a=1.8 k[m]$
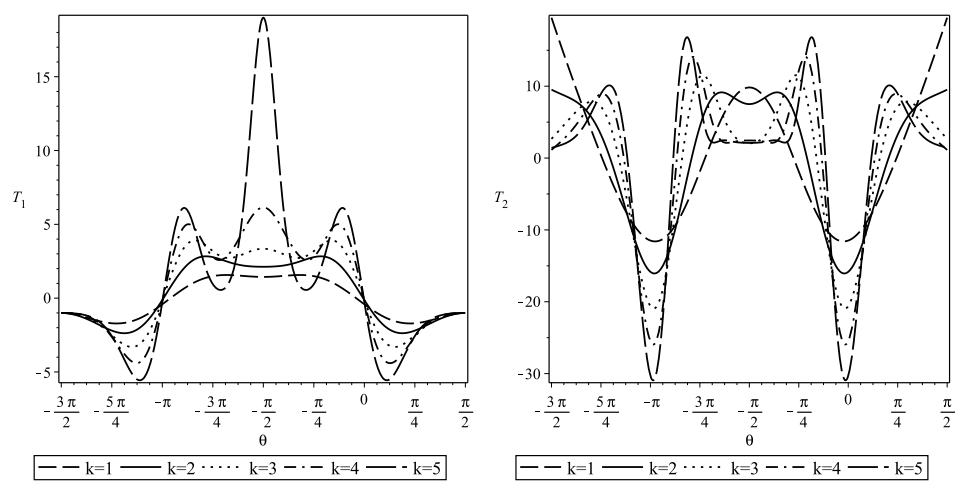

Figure 8: Left: Surface force $T_{1}$; Right: Surface force $T_{2}$. The torus radius is $a=1.8 k[\mathrm{~m}]$.

The figures indicate that all quantities such as bending moments, surface forces, shear force, and displacement are strongly effected by the radius ratio $\alpha=a / R$, and vary dramatically with $\theta$ both near to and far from the edge. 

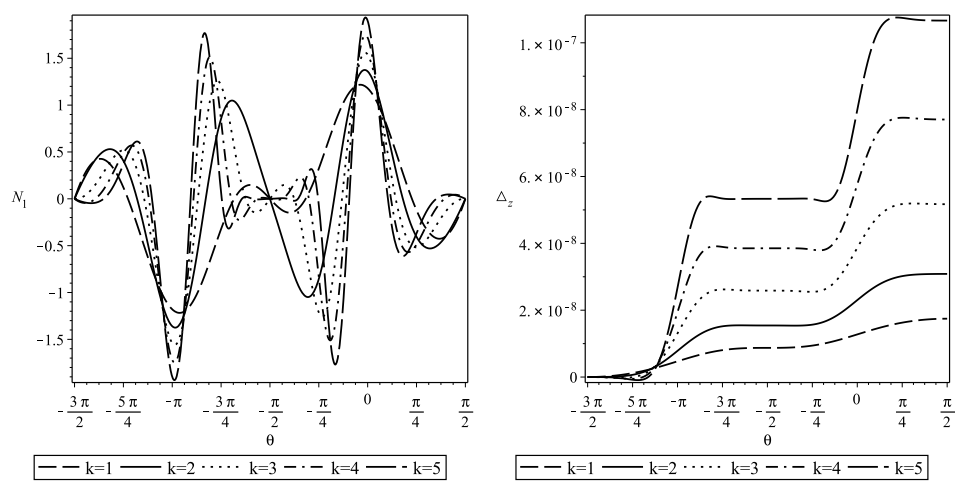

Figure 9: Left: Shear force $N_{1}$; Right: relative displacement in z direction $\triangle_{z}$. The torus radius is $a=1.8 k[m]$

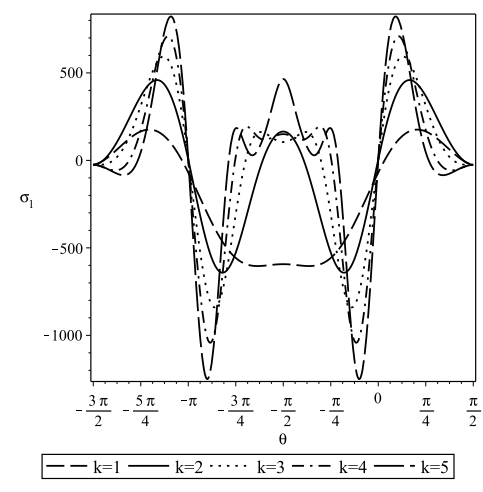

Figure 10: Stress $\sigma_{1}$

B. Complete torus with penetrate cut along the parallel $\theta=\frac{\pi}{2}$ and loaded with distributed bending moment $M_{0}$

Fig. 11 shows a complete torus with a penetrate cut along the parallel $\theta=\frac{\pi}{2}$ and loaded with distributed bending moment $M_{0}$. The boundary condition is:

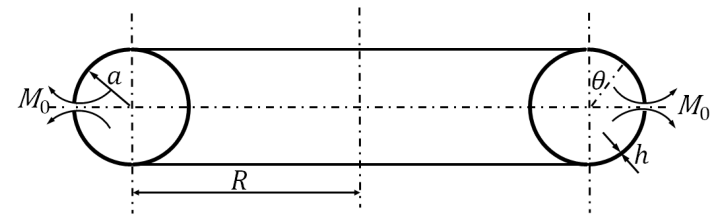

Figure 11: Torus with a cut along its parallel at $\theta=\frac{\pi}{2}$ or $\theta=-\frac{\pi}{2}$ under a pure moment $M_{0}$

$$
\begin{gathered}
\theta=\frac{\pi}{2}: T_{1}=0, N_{1}=0, M_{1}=-M_{0}, \\
\theta=-\frac{3 \pi}{2}: T_{1}=0, N_{1}=0, M_{1}=-M_{0}, \\
\theta=-\frac{3 \pi}{2}: \triangle_{z}=0 .
\end{gathered}
$$

For this problem, the constant $C$ can be determined by the boundary condition of $T_{1}$ at $\theta=\frac{\pi}{2}$, namely

$$
\theta=-\frac{\pi}{2}: T_{1}=0
$$

Taking into account the uniform distribution load $q=0$, the above equation gives $C=0$, and the boundary loading condition $T_{1}=0$ for both $\theta=\frac{\pi}{2}$ and $\theta=-\frac{3 \pi}{2}$ is satisfied. The rest of the boundary condition can be expressed in terms of functions 
$A(\theta)$ and $B(\theta)$, as follows:

$$
\begin{gathered}
\theta=\frac{\pi}{2}: B=0,-\frac{0.0001465201465}{(1+a) a^{2}} \frac{d A}{d \theta}=-M_{0}, \\
\theta=-\frac{3 \pi}{2}: B=0,-\frac{0.0001465201465}{(1+a) a^{2}} \frac{d A}{d \theta}=-M_{0}, \\
\theta=-\frac{3 \pi}{2}: \triangle_{z}=0 .
\end{gathered}
$$

The problem now becomes to solve $A(\theta)$ and $B(\theta)$ under conditions (63). The numerical results are shown in Fig. 12, 13, and 14 , which indicate that all quantities such as the bending moments, surface forces, shear force, and displacement are strongly affected by the radius ratio $\alpha=a / R$, and vary dramatically with the angle $\theta$ both near to and far from the edge.
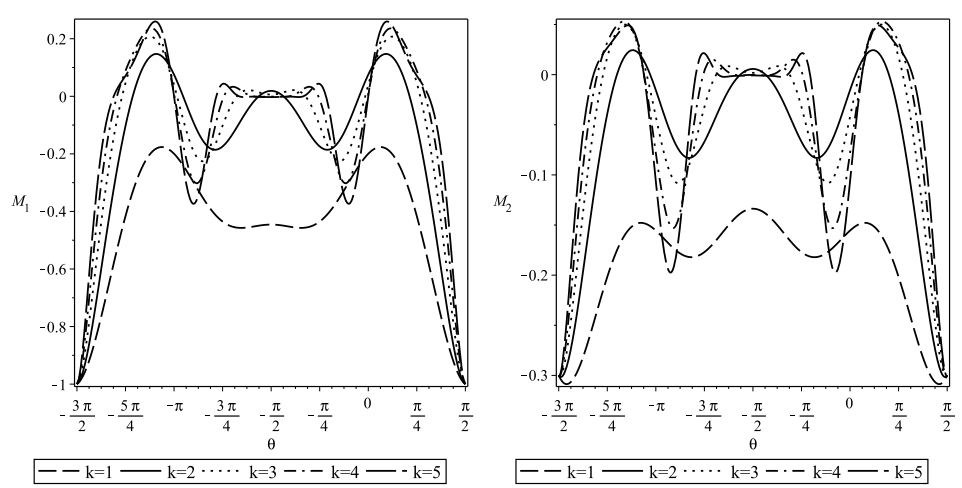

Figure 12: Left: Bending moment $M_{1}$; Right: Bending moment $M_{2}$. The torus radius is $a=1.8 k[m]$
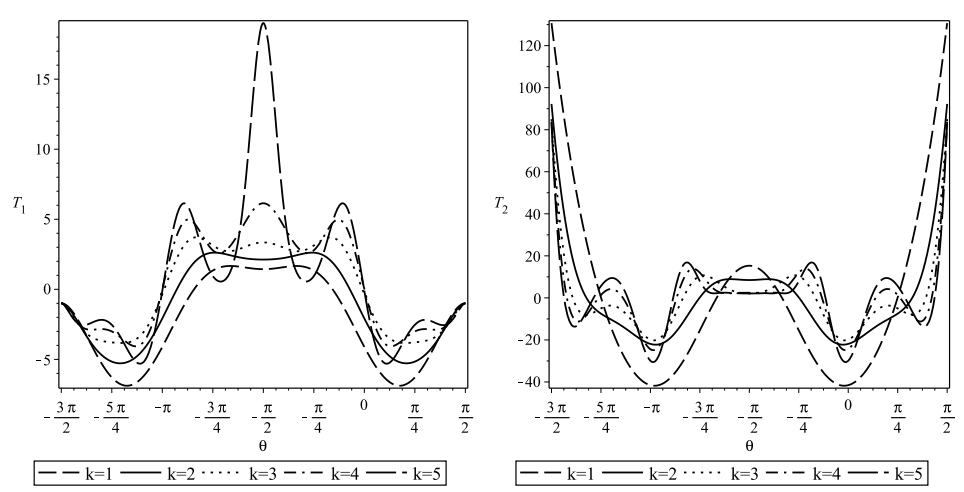

Figure 13: Left: Surface force $T_{1}$; Right: Surface force $T_{2}$. The torus radius is $a=1.8 k[\mathrm{~m}]$

\section{Half torus under edge vertical load along its parallel at $\theta=\frac{\pi}{2}$}

If we remove the inner region of a torus, we will have a half torus consisting of the outer region. This is under a vertical load along its parallel at $\theta=\frac{\pi}{2}$ and $\theta=\pi$, as shown in Fig. 15. The boundary condition is:

$$
\begin{aligned}
& \theta=0: T_{1}=0, N_{1}=-Q_{0}, M_{1}=0 \\
& \theta=\pi: T_{1}=0, N_{1}=-Q_{0}, M_{1}=0 \\
& \theta=\pi: \triangle_{z}=0
\end{aligned}
$$

For this problem, the constant $C$ can be determined by the boundary condition of $N_{1}$ at $\theta=0$, namely

$$
\theta=0: N_{1}=0 \text {. }
$$



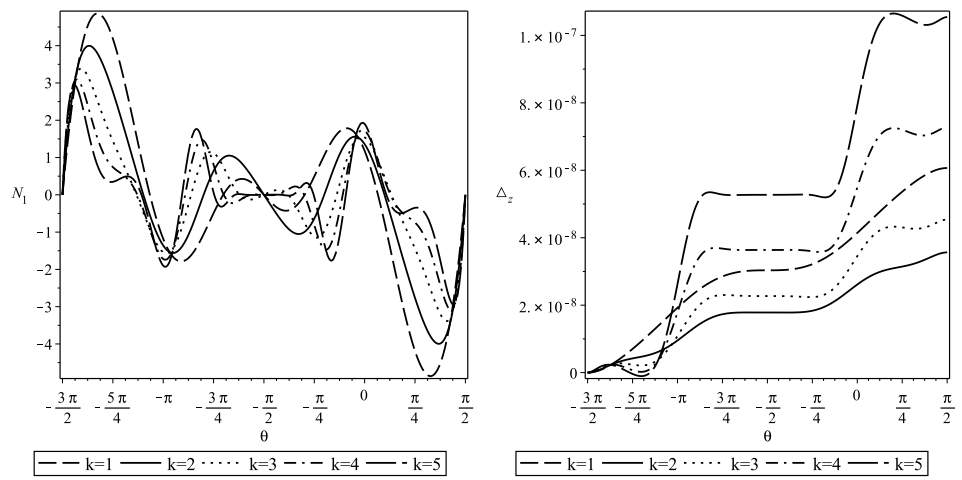

Figure 14: Left: Shear force $N_{1}$; Right: relative displace in z direction $\triangle_{z}$. The torus radius is $a=1.8 k[m]$

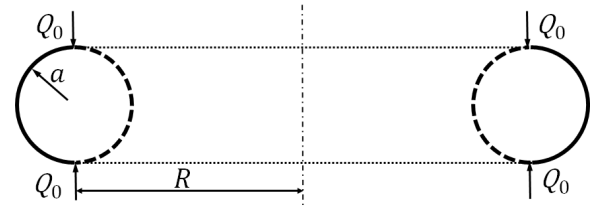

Figure 15: Torus with a cut along its parallel at $\theta=\frac{\pi}{2}$ or $\theta=-\frac{\pi}{2}$ under a force $Q_{0}$

Considering the uniform distribution load $q=0$, we obtain $C=\frac{Q_{0}}{7.565344159 a^{2}}$, in which case the boundary loading condition $N_{1}=0$ for both $\theta=0$ and $\theta=\Pi$ is satisfied. The rest of the boundary condition can be expressed in terms of $A(\theta)$ and $B(\theta)$, as follows:

$$
\begin{aligned}
& \theta=0: B+a=0,0.1025641026 a A-0.1465201465 \frac{d A}{d \theta}=0, \\
& \theta=\pi: B-a=0,0.1025641026 a A+0.1465201465 \frac{d A}{d \theta}=0 \\
& \theta=\pi: \triangle_{z}=0 .
\end{aligned}
$$

The problem now becomes to solve $A(\theta)$ and $B(\theta)$ under conditions (70). The numerical results are shown in Fig. 16, 17, and 18, and the stress is shown in Fig. 19. These figures indicate that all quantities, such as bending moments, surface forces, shear
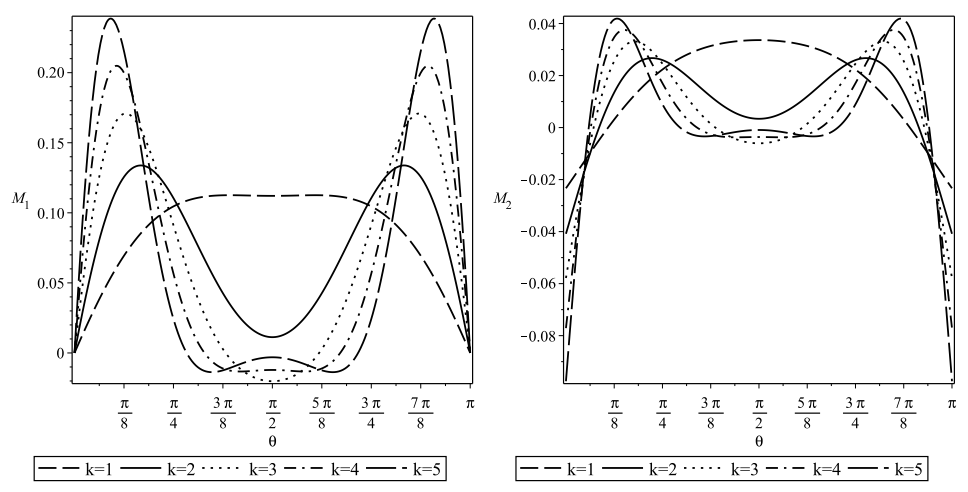

Figure 16: Left: Bending moment $M_{1}$; Right: Bending moment $M_{2}$. The torus radius is $a=1.8 k[\mathrm{~m}]$

force, and displacement, are strongly affected by the radius ratio $\alpha=a / R$, and vary dramatically with $\theta$ both near to and far from the edge.

\section{THE DIFFERNCE BETWEEN THE MEMBRANE THEORY AND BENDING THEORY OF TORUS}

In the history of theory of shells, a membrane theory is developed to simplify the analysis of shell structures. In the study 

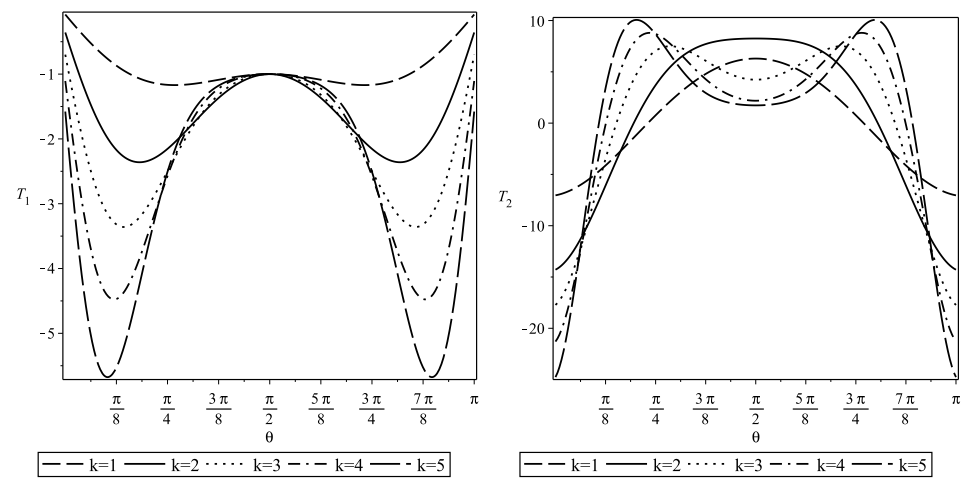

Figure 17: Left: Surface force $T_{1}$; Right: Surface force $T_{2}$. The torus radius is $a=1.8 k[\mathrm{~m}]$
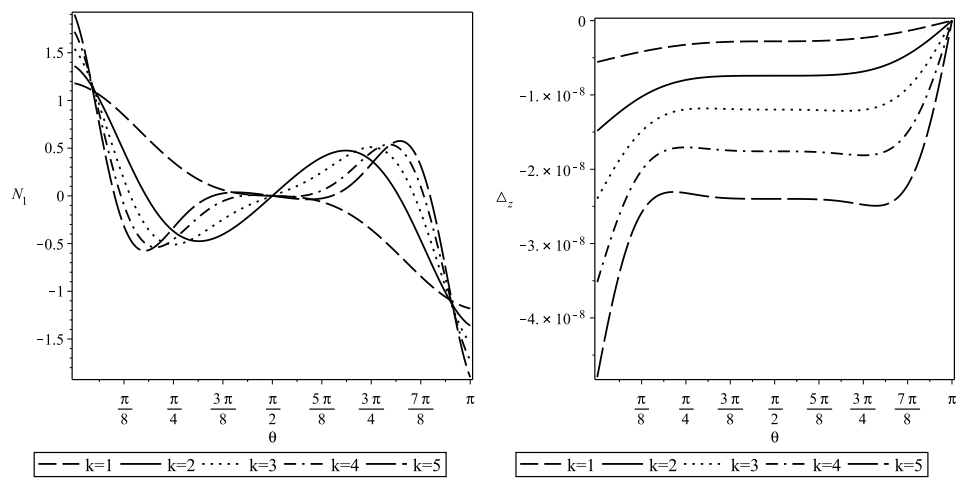

Figure 18: Left: Shear force $N_{1}$; Right: Relative displacement in z direction $\triangle_{z}$. The torus radius is $a=1.8 k[m]$

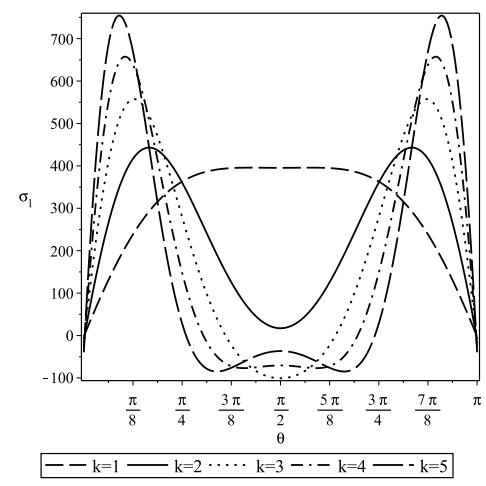

Figure 19: Stress $\sigma_{1}$

of the equilibrium of the shell element, this theory neglects all bending moments, which means that only surface forces $T_{1}, T_{2}$ are left. The membrane theory can make valuable predictions for shells with very small bending stiffness or when the changes of curvature and twist of the middle surface are very small. The above case studies show that membrane theory cannot provide reasonable results for a torus.

To obtain a clear picture of the difference between a membrane and the theory of a torus, let us perform comparisons for the above three case studies. For the case study in Section 5.1, the surface force is shown in Fig. 20. For the case study in Section 5.2, the comparison is shown in Fig. 21. For the case study in Section 5.3, the comparison is shown in Fig. 22. Fig. 20, 21, and 22 show that the results obtained from bending theory and membrane theory are totally different except at boundaries.

These comparison studies reveal that the results from the membrane theory of the torus are questionable. Therefore, the bending theory of shells should be used for the analysis of a torus. 


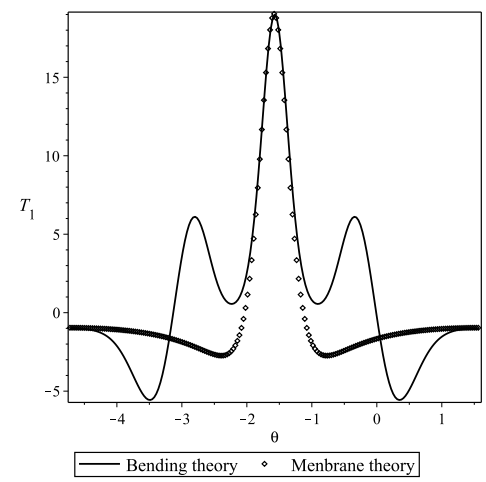

Figure 20: Bending theory (solid line) and membrane theory (point). Surface force $T_{1}$ for torus radius $a=0.9[\mathrm{~m}]$

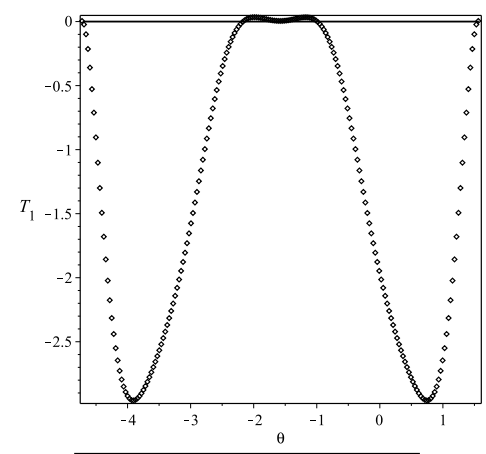

Figure 21: Bending theory (point) and membrane theory (solid line). Surface force $T_{1}$ for torus radius is $a=0.9[\mathrm{~m}]$

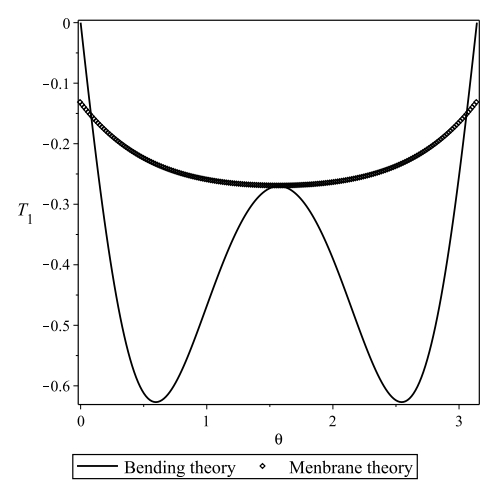

Figure 22: Bending theory (point line) and membrane theory (solid line). Surface force $T_{1}$ for torus radius is $a=0.9[\mathrm{~m}]$

\section{VERIFICATION OF OUR RESULTS BY FINITE ELEMENT ANALYSIS}

To verify our result, we carried out a finite element analysis for the case study in Section 5.1. The comparisons are shown in Fig. 23. The finite element results were simulated by ABAQUS with shell element S4R. We applied the boundary condition $\triangle_{z}(-3 \pi / 2)=0$ to cancel the rigid body motion, which might be the reason for minor differences in the range $\theta \in[-3 \pi / 2,-5 \pi / 4]$. From physics point of view, our result is symmetric respect to the axes of $\theta=-\pi / 2$ while the FEA has slightly symmetry breaking. Therefore, our results are more trustable since the finite element analysis supports our numerical prediction nicely. 

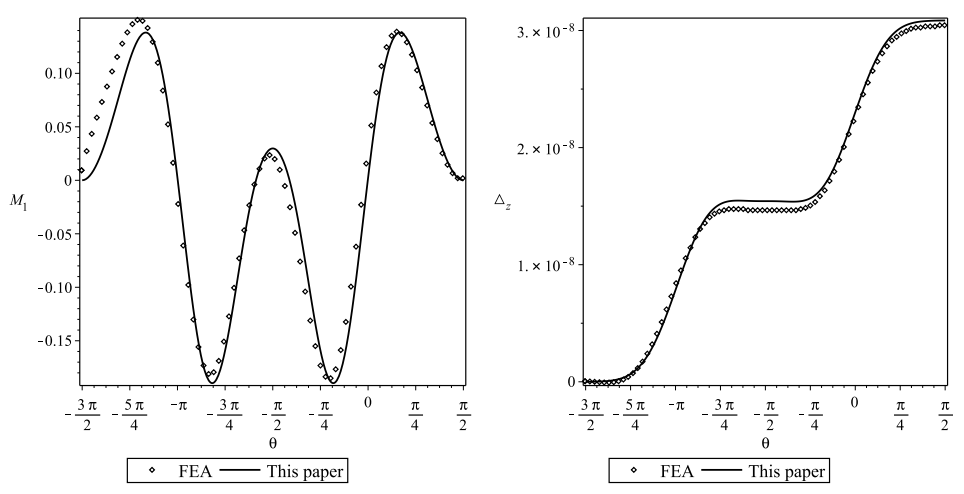

Figure 23: Results for case study in Section 5.1, as shown in Fig. 6. Left: Bending moment $M_{1}$; Right: Vertical displacement $\triangle_{z}$. The torus radius is $a=0.36[\mathrm{~m}]$

\section{CONCLUSIONS AND FUTURE PERSPECTIVES}

We have obtained an exact solution of the complex-form ODE for the symmetrical deformation of a torus. This solution is expressed by the Heun functions. However, as we stated, the Huen function cannot be split into real and imaginary parts explicitly. To solve this issue, we proposed a new strategy of transforming the complex-form ODE of torus into an rela-form ODE in terms of $A(\theta), B(\theta)$, and $\triangle_{z}(\theta)$, which allowed us to easily deal with different kinds of boundary conditions.

To verify our formulation, we wrote a computational code in Maple and carried out some numerical simulations. The validation of our numerical results was confirmedand supported by the finite element analysis. The numerical investigation shows that the bending theory of shells should be used for torus deformation analysis.

Regarding the future perspectives, it must be pointed out that the complex-form governing ODE of a torus has some disadvantages, for instance, the the complex-form governing ODE cannot be used to deal with dynamical and buckling problems. This definitely limits the use of complex-form modeling. To overcome this shortcoming, displacement-type ODE of shells must be used [20], which remains an open problem [20].

Acknowledgement: The author is honored to have benefites from my supervisor Prof. Dr.Ing Wei Chang (W. Zhang), it is my privilege to dedicate this paper to the memories of Prof. Zhang for his great contribution to the theory of the thin torus. The author appreciates my student Mr. Guang-Kai Song, for preparing Figures 1-4, 6, 11, 15 and provided finite element analysis data for Figure 23. I also wish to express my gratitudes to anonymous reviewers for their high-level academic comments that helps me to enhance the quality of this paper.

Conflict of interest The author declares that he has no known competing financial interests or personal relationships that could have appeared to influence the work reported in this paper.

Data avalibility The data that support the findings of this study are available from the corresponding author upon reasonable request.

[1] G. Weihs. Über Spannungs- und Formänderungszustände in dünnen. Hohlreifen. Halle a. S. 1911.

[2] H. Reissner, Spannungen in Kugelschalen (Kuppeln). Festschrift Heinrich Müller-Breslau (A. Kröner, Leipzig, 1912), pp.181-193

[3] E. Meissner,Über und Elastizitat Festigkeit dunner Schalen, Viertelschr. D. nature.Ges., Bd.60, Zurich (1915).

[4] H. Wissler, Festigkeiberechung von Ringsflachen, Promotionarbeit, Zurich (1916). https://doi.org/10.3929/ethz-a-000099037

[5] F. Tölke, Ingenieur Archiv., 9 (1938), 282

[6] W. Chang (W. Zhang), Derspannungszustand in kreisringschale und ähnlichenSchalen mit Scheitelkreisringen unter drehsymmetrischer Belastung, Arbeitzur Erlangung des Grades eines Doctor-Ingenieurs der Technichen Hochschule, Berlin, 1944.(English translation: Zhang, W., Toroidal shells, Sci. Rep Nat. Tsinghua Univ., Ser A. 259-349 (1949))

[7] E. Reissner, On bending of curved thin-walled tubes, Proc. National Academy of Sci., (1949)36, 204-208.

[8] R.A. Clark, R.A. and E. Reissner, Bending of curved tubes, Advances in Applied ;Hechanics, vol. II, Academic Press (1950)

[9] R.A. Clark, On the theory of thin elastic toroidal shells, J. Mech. Phys. Solids,29(1950)3:146-178.

[10] N.C. Dahl, Toroidal-shell expansion joints, J. of Applied Mechanics, ASME,20(1953):497-503.

[11] V.V. Novozhilov, The Theory of Thin Shell. (Noordhoff, Groningen, 1959).

[12] L.N. Tao, On toroidal shells, J. of Math and Physics, 38,(1959):130-134.

[13] $C_{i} R_{i}$ Steele, Toroidal pressure vessels, J. Spacecr. Rocket., 2(1965):937-943,

[14] W.Z. Qian and S.C. Liang, Complex form equation and asymptotic solution, J. of Tsinghua University, 19(1979)(1): 27-47, 
[15] Z.H. Xia and W. Zhang, The general solution for thin-walled curved tubes with arbitrary loadings and various boundary conditions. Int. J. Pressures and Piping 26(1986):129-144.

[16] W. Zhang, W.M. Ren and B.H. Sun, Toroidal Shells - history, current situation and future, Fifth Conf. of Space Structures, Lanzhou, China 1990.

[17] R.J. Zhang and W. Zhang, Turning point solution for thin toroidal shell vibrations. Int. J. Solids Structures 27(1991)(10):1311-1326.

[18] R.J. Zhang and W. Zhang, Toroidal shells under Nonsymmetric Loading, Int. J. Solids Structures, 31(1994)19:2735-2750.

[19] B. Audoly and Y. Pomeau, Elasticity and Geometry - From hair curls to the non-linear response of shells. University of Cambridge, Cambridge, 2010

[20] B.H. Sun, Closed-form solution of axisymmetric slender elastic toroidal shells, Journal of Engineering Mechanics, 136(2010) 10:12811288.

[21] B.H. Sun, Toroidal Shells, (Nova Novinka, New York, 2012)

[22] B.H. Sun, Centenary studies of toroidal shells and in memory of Prof. Zhang Wei, Mechanics in Engineering, 37(2013)(3). (In Chinese)

[23] B.H. Sun, Exact solution of Qian's equation of slender toroidal shells. Mechanics in Engineering, 38(2018)(5):567-569.(in Chinese)

[24] A. Ronveaux, Heun's Differential Equations. Oxford University Press, 1995.

[25] https://en.wikipedia.org/wiki/Heun_function

[26] J.E. Marsden, L. Sirovich and S.S. Antman eds. Hypergeometric Functions and Their Applications. Texts in Applied Mathematics. 56. New York: Springer-Verlag, 1991.

[27] V.V. Kuznetsov and S.V. Levyakov, Nonlinear pure bending of toroidal shells of arbitrary cross-section. International Journal of Solids and Structures, 38(2001) 40-41:7343-7354.

[28] A. Zingoni, N. Enoma and N. Govender, Equatorial bending of an elliptic toroidal shell. Thin-Walled Structures, 96(2015):286-294.

[29] W. Jiammeepreecha and S. Chucheepsakul, Nonlinear static analysis of an underwater elastic semi-toroidal shell. Thin-Walled Structures, 116(2017): 12-18.

[30] N. Enoma and A. Zingoni (2020). Analytical formulation and numerical modelling for multi-shell toroidal pressure vessels. Computers \& Structures, 232(2020), Article 105811.

[31] Marple https://www.maplesoft.com/ 